\title{
Tromboembolismo pulmonar en pacientes hospitalizados entre 1994-2000: serie de autopsia
}

\author{
M. R. BURÓN FERNÁNDEZ, E. PINTOR HOLGUÍN', P. MÍNGUEZ GARCÍA, \\ J. A. NUEVO GONZÁLEZ, J. J. PUCHE PANIAGUA, C. GÓMEZ SÁNCHEZ- \\ BIEZMA, P. ARAGONCILLO BALLESTEROS ${ }^{2}$
}

Servicios de Medicina Interna $y^{2}$ Anatomía Patológica. Hospital Clínico San Carlos. ${ }^{1}$ Departamento de Especialidades Médicas Aplicadas. Universidad Europea. Madrid

PULMONARY THROMBOEMBOLISM IN HOSPITALIZED PATIENTS

DURING THE PERIOD 1994-2000: AN AUTOPSY STUDY

\section{RESUMEN}

Introducción: El tromboembolismo pulmonar (TEP) ha sido durante muchos años una causa muy importante de morbi-mortalidad en los pacientes hospitalizados. El empleo de forma profiláctica de Heparinas de Bajo Peso Molecular (HBPM) en pacientes ingresados en la última década del siglo XX parece haber disminuido de forma significativa su incidencia clínica. Pretendemos ver si el empleo de HBPM como medida de tromboprofilaxis ha modificado el hallazgo de esta patología en autopsias.

Material y métodos: Estudio retrospectivo de todas las autopsias realizadas en adultos en el Hospital Clínico San Carlos (Madrid) en un periodo de 6 años (Enero 1994-Diciembre 1999). Se incluyeron en el estudio todas aquellas que presentaron hallazgos anatomopatológicos (AP) de TEP y se rellenó un protocolo en el que se incluyeron datos AP, datos epidemiológicos, clínicos y terapéuticos.

Resultados: En el periodo estudiado se realizaron 483 necropsias, de las cuales $40(8,3 \%)$ tenían datos de TEP. La mayor parte de ellos tenían más de 50 años (85\%) y entre los factores de riesgo destacaron el reposoencamamiento, la enfermedad crónica médica y la presencia de tumores malignos (adenocarcinomas). Sólo se sospechó el diagnóstico pre-mortem en $5(12,5 \%)$ y $15(37,5 \%)$ desarrollaron la ETE a pesar de haber recibido tratamiento profiláctico con HBPM.

Conclusiones: El TEP continúa siendo una causa muy importante de mortalidad de los pacientes ingresados en el hospital. El aumento de la esperanza de vida, de la supervivencia de enfermedades crónicas médicas y tumorales determina que hay que tener muy en cuenta esta patología. El empleo de una HBPM como profilaxis no excluye la posibilidad de esta entidad.

PALABRAS CLAVE: Autopsia. Tromboembolismo pulmonar. HBPM.

\begin{abstract}
Background: Pulmonary thromboembolism (PTE) has been for a long time a significant cause of morbidity and mortality in hospitalized patients. The utility of Low-Molecular Weight Heparins (LMWH) in these patients in the last decade of the XX century has decreased the incidence of this disease.

We try to know if the massive useful of LMWH as thromboprophylasis is diminishing its incidence in autopsies.

Material y methods: Retrospective study of all the autopsies in adults in the Hospital Clínico San Carlos (Madrid) in a period of 6 years (from January 1994 to December 1999). There were reviewed those necropsies which had pathological data of pulmonary thromboembolism and several items were studied: anatomopathological, epidemiological, clinical and therapeutical.

Results: 483 necropsies were performed in this period; 40 (8.3\%) had PE. Most of them were older than 50 years (85\%) and the most important risk factors associated were bedridden, chronic cardiovascular diseases and malignant neoplasias. Only Pre-mortem diagnosis was only suspected in 5 patients (12.5\%) and 15 of them (37.5\%) had a fatal pulmonary embolism despite receipt of thromboprophylasis with LMWH.

Conclusions: PTE is still an important cause of mortality in hospitalized patients. The increased of life expect, survival of chronic cardiovascular and malignant disease made PTE a frequent possibility situation in hospitalized patients. Receipt of LMWH as thromboprophylaxis is not always effective to avoid PTE
\end{abstract}

KEY WORDS: Autopsy. Pulmonary thromboembolism. LMWH.

Burón Fernández, MR, Pintor Holguín E, Mínguez García P, Nuevo González J A, Puche Paniagua J J, Gómez. Sanchez-Biezma C, Aragoncillo Ballesteros P. Tromboembolismo pulmonar en pacientes hospitalizados entre 1994-2000: serie de autopsia. An Med Interna (Madrid) 2006; 23: 317-320. 


\section{INTRODUCCIÓN}

La enfermedad tromboembólica (ETE) ha sido y es una patología frecuente a nivel hospitalario; tanto como causa de ingreso como causa de importante morbi-mortalidad en los pacientes ingresados por otros motivos (1). Su incidencia real es difícil de estimar ya que su diagnóstico suele basarse primero en la sospecha clínica y después en pruebas que tienen una sensibilidad y especificidad limitada. La variedad e inespecificidad del cuadro clínico, con formas asintomáticas u oligosintomáticas y en el extremo opuesto, muertes súbitas cuya causa queda sin conocer de no realizarse el estudio necrópsico hace que su incidencia real no esté bien establecida.

El tromboembolismo pulmonar (TEP) ha sido estimado como un $5 \%$ de causas de muerte o aproximadamente 60.000 muertes al año en EE.UU. (2-5). En los pacientes con TEP la supervivencia aumenta si se establece el diagnóstico y comienza el tratamiento, sin embargo sólo un $30 \%$ de los casos de embolia de pulmón son diagnosticados antes de la muerte.

La incidencia de esta entidad podría haber cambiado en la última década: por un lado podría haber aumentado ya que los factores de riesgo de ETE podrían ser más frecuentes en los pacientes hospitalizados (por envejecimiento de la población) (6), sin embargo por otro lado la utilización amplia de heparinas de bajo peso molecular (HBPM) como medidas profilácticas (7) tanto de los enfermos con problemas "médicos" como con problemas "quirúrgicos" podría haber hecho que descendiera el hallazgo AP de TEP.

Pretendemos ver por tanto si se ha modificado la incidencia de TEP como hallazgo de autopsia, si han variado los factores de riesgo en los pacientes en los que se encuentra y cuanto de efectiva ha sido la tromboprofilaxis con HBPM.

\section{MATERIAL Y MÉTODOS}

Se ha realizado un estudio retrospectivo de todas las autopsias realizadas en adultos en el Hospital Clínico Universitario San Carlos de Madrid (HCUSC), en un periodo de seis años, entre enero de 1994 y diciembre de 1999.

Se incluyeron en este estudio, todas aquellas en las que en el estudio necrópsico se encontraron hallazgos anatomopatológicos característicos de TEP.

Los hallazgos fueron clasificados en función del tipo de arterias afectadas en:

1. TEP severo, cuando afectaba al menos a dos arterias lobares,

2. TEP moderado cuando afectaba a una arteria lobar $y / o$ varias segmentarias,

3. TEP leve cuando afectaba pocas arterias segmentarias.

Por cada paciente se cumplimentó un protocolo donde se incluyen parámetros epidemiológicos (edad, peso, sexo), clínicos (factores de riesgo para el desarrollo de ETE entre los cuales se incluyeron: el reposo o permanencia en cama, presencia de tumor conocido (el tipo y la localización aparecido en necropsias), cirugías previas recientes, enfermedades crónicas que favorecen situaciones de hipercoagula- bilidad (insuficiencia cardiaca congestiva, EPOC, IAM reciente); la presencia de síntomas (disnea, hemoptisis y dolor torácico), junto con la presencia de gasometría arterial compatible, índice de sospecha clínica y terapéuticos (profilaxis o tratamiento con anticoagulantes).

\section{RESULTADOS}

Durante el periodo comentado se realizaron 483 necropsias a adultos de los cuales $40(8,3 \%)$ tenían datos anatomopatológicos de TEP. Según los hallazgos anatomopatológicos en un $70 \%$ el TEP fue la causa fundamental de muerte, un $22,5 \%$ se encontró como factor coadyuvante y en un 7,5\% fue únicamente un hallazgo casual.

Separando los datos por años se encontraron ligeras variaciones en la incidencia oscilando entre el 5,4-13,8\% (Fig. 1).

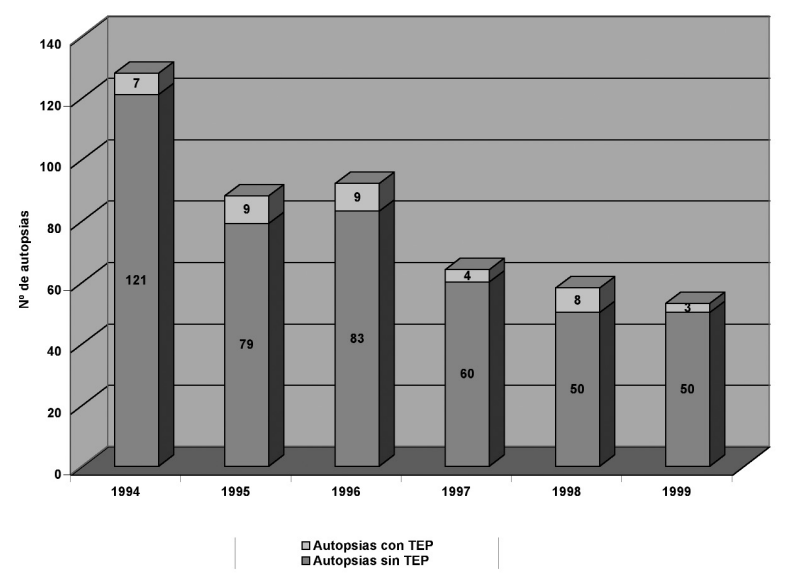

Fig. 1. TEP en autopsias en adultos entre 1994-2000.

De los 40 pacientes del estudio un $62,5 \%$ fueron varones frente a un $37,5 \%$ de mujeres, cuya edad media fue de 65 años y la mayor parte de ellos (85\%) eran mayores de 50 años.

En el análisis de los factores de riesgo se encontró: un $82,5 \%$ con reposo o encajamiento prolongado, un $47,5 \%$ con enfermedad médica crónica (EPOC, ICC e IAM previo), un $45 \%$ con enfermedad tumoral, y un $22,5 \%$ postquirúrgicos. De los 18 pacientes (45\%) que presentaron enfermedad tumoral en $10(25 \%)$ era conocido y en $8(20 \%)$ fue un hallazgo en la necropsia. De ellos los más frecuentes fueron adenocarcinoma de pulmón (22\%), adenocarcinoma de colon $(22 \%)$ y adenocarcinoma gástrico (17\%).

La sospecha clínica pre-mortem se realizó en un 12,5\% del total de pacientes. Entre los síntomas analizados un $75 \%$ presentaron disnea, un $20 \%$ dolor torácico y un $10 \%$ hemoptisis. Se objetivó una gasometría arterial basal compatible con TEP en un $45 \%$ de los casos.

Por último se tuvo en cuenta los pacientes que estaban en tratamiento profiláctico con heparina de bajo peso molecular 
ajustada dosis según peso, un 37,5\% desarrollaron TEP a pesar de recibir HBPM.

\section{DISCUSIÓN}

Aunque desde mediados del siglo pasado se ha planteado la posibilidad de utilizar anticoagulantes para evitar la ETE en pacientes sometidos a cirugía, no ha sido hasta la últimas décadas del siglo XX donde se ha ido estandarizando su empleo.

La aparición de las HBPM (anticoagulantes eficaces con poco riesgo, sin necesidad de controles de coagulación y fácilmente administrables) en la década de los 90, ha determinado el empleo masivo de este grupo de fármacos tanto en la profilaxis como en el tratamiento de la ETE (8). La utilización profiláctica de esta medida en pacientes de riesgo (tanto quirúrgicos como médicos) parece haber determinado un cambio en la incidencia de esta patología en pacientes hospitalizados. Sin embargo también hay que tener en cuenta que los pacientes ingresados, en especial los no-quirúrgicos tienen más factores de riesgo para ETE que hace años.

El TEP sigue constituyendo una entidad frecuente como hallazgo de autopsia. En nuestro estudio supuso un 8,3\% del total. El hallazgo en autopsias ha ido disminuyendo progresivamente desde los años 60 donde alcanzaba un $35 \%$ hasta los noventa donde descendió hasta un 10-20\% (9-11), pero que se ha mantenido entre el $5-10 \%$ en las series que han recogido casos en los últimos 15 años (12-14) y de ese porcentaje en al menos en la mitad fue la causa fundamental de la muerte $(15,16)$.

En los últimos 10-15 años, aunque la cifra total de TEP diagnosticada en autopsias no parece haber disminuido de forma muy significativa (17), si que se ha modificado la importancia de los factores de riesgo.

Mientras que la introducción de tromboprofilaxis farma- cológica, si ha modificado la incidencia de ETE en los pacientes quirúrgicos $(18,19)$, no parece que el efecto haya sido similar en los pacientes "médicos" (20). Así en las series recientes de autopsia donde se objetivó tromboembolismo pulmonar, solo una cuarta parte de los pacientes tenían antecedentes quirúrgicos, dato que se mantiene también en nuestro estudio $(22,5 \%)$. En los pacientes no quirúrgicos, las enfermedades cardiovasculares crónicas, el encamamiento $(15,16)$, la edad (21) y los tumores malignos (22) son las causas predominantes. Muchos de ellos presentaban varios de estos factores simultáneamente. Datos similares han sido publicados en estudios recientes (11-13).

El TEP continúa siendo de difícil diagnóstico a pesar de constituir una de las causas más frecuentes de muerte en pacientes hospitalizados $(1,10,12,23)$. La sospecha premortem en la mayor parte de los estudios es menor del $30 \%$ y es especialmente difícil en los pacientes con patología cardiopulmonar crónica $(24,25)$.

La utilización de tromboprofilaxis en las últimas décadas ha disminuido las complicaciones trombóticas venosas de los pacientes ingresados. Sin embargo, hay pacientes que a pesar de recibir medidas preventivas tienen ETE que incluso puede llegar a ser mortal $(26,27)$. Según un reciente estudio (20), la utilización de HBPM en pacientes hospitalizados no quirúrgicos como medida profiláctica no disminuye la mortalidad, aunque si disminuyó el hallazgo de TEP en las autopsias sin llegar a ser estadísticamente significativo.

Por tanto concluimos que el TEP continúa siendo un hallazgo frecuente en las autopsias de pacientes con patología médica hospitalizados a pesar del empleo de HBPM como medida profiláctica. Asimismo el índice de sospecha de esta entidad sigue siendo muy bajo.

A los enfermos de alto riesgo (con varios factores de riesgo para ETE) debería valorarse dosis más altas de HBPM, siendo muy interesante la realización de estudios prospectivos randomizados para identificar el efecto de la profilaxis a altas dosis en este grupo de pacientes como ya ha sido apuntado en otros estudios (28).

\section{Bibliografía}

1. Stein PD, Beemath A, Olson RE. Trends in the incidence of pulmonary embolism and deep venous thrombosis in hospitalized patients. Am J Cardiol 2005; 95: 1525-6.

2. Timothy I, Morgenthaler, Ryu JH. Clinical characteristics of fatal pulmonary enbolism in a referral hospital. Mayo Clin Proc 1995; 70: 417-424.

3. Dismuke SE, Wagener EH. Pulmonary embolism as a cause of death: the changing mortality in hospitalized patients. JAMA $1986 ; 255$ : 2039-2042.

4. Goldhaber SZ, Hennekens CH. Time trends in hospital mortality and diagnosis of pulmonary embolism. Am Heart J 1982; 104: 305-306

5. Dalen JE, Alpert JS. Natural history of pulmonary embolism. Prog Cardiovasc Dis 1975; 17: 259-270.

6. Silverstein MD, Heit JA, Mohr DN, Petterson TM, O'Fallon WM, Melton LJ 3rd. Trends in the incidence of deep vein thrombosis and pulmonary embolism: a 25-year population-based study. Arch Intern Med 1998; 158: 585-93.

7. Kaboli PJ, Brenner A, Dunn AS. Prevention of venous thromboembolism in medical and surgical patients. Cleve Clin J Med 2005 ; 72 : S7-13.
8. Merli GJ. Pulmonary embolism in medical patients: improved diagnosis and the role of low-molecular-weight heparin in prevention and treatment. J Thromb Thrombolysis 2004 ; 18: 117-25.

9. Nordstrom M, Lindblad B . Autopsy-verified venous thromboembolism within a defined urban population-the city of Malmo, Sweden.APMIS 1998; 106: 378-84.

10. Lindblad B, Sternby NH, Bergquist D. Incidence of venous thromboembolism verified by necropsy over 30 years. BMJ 1991; 302: 709711.

11. Golin V, Sprovieri SR, Bedrikow R, Salles MJ. Pulmonary thromboembolism: retrospective study of necropsies performed over 24 years in a university hospital in Brazil. Sao Paulo Med J. 2002 ; 120: $105-8$.

12. Alikhan R, Peters F, Wilmott R, Cohen AT. Fatal pulmonary embolism in hospitalised patients: a necropsy review. J Clin Pathol 2004; 57: 1254-7.

13. Spiliopoulou C, Papadodima S, Kotakidis N, Koutselinis A. Clinical diagnoses and autopsy findings: a retrospective analysis of 252 cases in Greece. Arch Pathol Lab Med 2005 ; 129: 210-4. 
14. Liu HS, Kho BC, Chan JC, Cheung FM, Lau KY, Choi FP, Wu WC, Yau TK. Venous thromboembolism in the Chinese population--experience in a regional hospital in Hong Kong. Hong Kong Med J 2002; 8: 400-5

15. Baglin TP, White K, Charles A. Fatal pulmonary embolism in hospitalised medical patients. J Clin Pathol 1997; 50: 609-610.

16. Rubinstein I, Murray D, Moffstein V. Fatal pulmonary emboly in hospitalized patients. An autopsy study. Arch Intern Med 1988; 148: $1425-1426$.

17. Colantonio D, Casale R, Lorenzetti G, Pasqualetti P. Fatal pulmonary thromboembolism: is its incidente increasing or diminishing? Analysis of 178 cases from 1971 to 1988 . Cardiologia 1989; 34: 563-5.

18. Pezzuoli G, Neri Serneri GG, Settembrini PG, Coggi G, Olivari N, Negri G,et al. Effectiveness and safety of the low-molecular-weight heparin CY 216 in the prevention of fatal pulmonary embolism and thromboembolic death in general surgery. A multicentre, doubleblind, randomized, controlled clinical trial versus placebo (STEP). STEP Study Group. Haemostasis 1990; 20 (Supl. 1): 193-204.

19. Haas S, Wolf H, Kakkar AK, Fareed J, Encke A. Prevention of fatal pulmonary embolism and mortality in surgical patients: a ramdomized double-blind comparison of LMWH with unfractionated heparin. Thromb Haemost 2005; 94: 814-9.

20. Mahe I, Bergmann JF, d'Azemar P, Vaissie JJ, Caulin C. Lack of effect of a low-molecular-weight heparin (nadroparin) on mortality in bedridden medical in-patients: a prospective randomised double-blind study. Eur J Clin Pharmacol 2005; 61: 347-51

21. Masotti L, Ceccarelli E, Cappelli R, Guerrini M, Forconi S. Pulmonary embolism in the elderly: clinical, instrumental and laboratory aspects. Gerontology 2000; 46: 205-2011

22. García-Rodeja ME, Gamallo C, Arribas JR, Monereo A. Importancia de los factores de riesgo en la extensión del tromboembolismo pulmonar. Med Clin (Barc) 1990; 95: 92-94.

23. Stein PD, Henry JW. Prevalence of acute pulmonary embolism among patients in a general hospital and at autopsy. Chest 1995; 108: 978-81.

24. Pineda LA, Hathwar VS, Grant BJ. Clinical suspicion of fatal pulmonary embolism.Chest 2001; 120: 791-795.

25. Arribas JR, Arnalich F, García-Rodeja ME, Monereo A, Lahoz C, Gamallo C, et al. ¿Qué factores hacen que el diagnostico de tromboembolismo pulmonary sea difical? Med Clin (Barc) 1990; 94: 525-7.

26. Patel R, Cook DJ, Meade MO, Griffith LE, Metha G, Rocker GM, et al. Burden of illness in venous thromboembolism in critical care: a multicenter observational study. J Crit Care 2005; 20: 341-7

27. Kakkar AK, Haas S, Wolf H, Encke A. Evaliation of perioperative fatal pulmomary embolism and death in cancer surgical patients: the MC-4 cancer substudy. Thromb Haemost 2005; 94: 867-71

28. Prandoni P, Sabbion P, Tanduo C, Errigo G, Zanon E, Bernardi E. Prevention of venous thromboembolism in high-risk surgical and medical patients. Semin Vasc Med 2001; 1: 61-70. 